\title{
PREVISION DES SURCOTES DANS L'ESTUAIRE EXTERNE DE LA LOIRE PAR UNE APPROCHE STATISTIQUE
}

\author{
H. Ghadri ; O. A. Khalifa ; A. Grovel \\ Laboratoire de Génie Civil, E.N.S.M. Nantes \\ L.U.T. Saint-Nazaire, BP: 420,44606 Saint-Nazaire Cedex
}

\begin{abstract}
Nowadays the methods of prediction of the tide on the coasts of Europe use a harmonic formula in which harmonic constants dependant on every site intervene. In this formula the generating forces taken into account proceed essentially with the periodic movements of stars.

But, rapidly it appears that local meteorologic phenomena disturb these predictions. Important damages at the time of a storm in the channel and the north sea on the maritime works are due to the unexpected elevation of the sea level. Now these phenomena of tidal overelevation become in the context of the coastal safeguard.

There are many determinating methods and they have been studied in France, one by FREROT (1988). Initially our approach of the phenomena is statistical and therefore it relies on the comparative study of on one hand the harmonic analysis of this site, and on the other hand a set of measures of the real tide and the measured wind in the station of Montoir de Bretagne.

This comparative study will permit to establish semi-empiric laws for this particular site and possibly consider their generalisation for other sites.
\end{abstract}

\section{RESUME}

Actuellement les méthodes de prédiction de la marée sur les côtes d'Europe utilisent une formule harmonique faisant intervenir des constantes harmoniques dépendant de chaque lieu. Dans cette formule, les forces génératrices prises en compte proviennent essentiellement des mouvements périodiques des astres.

Mais, il est apparu rapidement que des phénomènes météorologiques locaux perturbent fortement ces prédictions. D'importants dégâts constatés lors de tempêtes en Manche et en Mer du Nord sur des ouviages maritimes (franchissement de digues) sont dûs à des élévations du niveau de la mer non prévues. Ces phénomènes de surcote reviennent à l'ordre dı jour, dans le contexte de volonté de sauvegarde du littoral.

L'un des facteurs prépondérants à l'origine de ces surcotes est le vent. L'objectif de notre étude est de déterminer ces surcotes dans l'estuaire externe de la Loire.

Plusieurs méthodes déterministes existent et ont été étudiées en France dont une par O. FREROT. Notre approche du phénomène est dans un premier temps statistique et repose par conséquent sur l'étude comparative d'une part de l'analyse harmonique sur ce site et d'autre part d'un ensemble de mesures de la marée réelle, (marégraphe du Port Autonome de Nantes-St-Nazaire) et du vent mesuré à la station de Montoir de Bretagne.

Cette étude comparative permettra de dégager des lois semi-empiriques pour ce site et envisager éventuellement leur généralisation à d'autres lieux. 


\section{MOTS CLES :}

Marée / Surcote / Tempête / Météorologie / Pression atmosphérique / Vent / Littoral.

\section{INTRODUCTION}

Pour la conception et le dimensionnement en hauteur des ouvrages non soumis à la houle, l'ingénieur de génie civil devra déterminer le niveau des plus hautes mers possibles, quelle que soit son origine (astronomique ou météorologique). En revanche, quand il s'agit de creuser un chenal et d'en déterminer la profondeur pour l'entrée des navires d'un tirant d'eau donné, c'est le niveau des plus basses mers possibles qui intéresse le concepteur.

Sur un ouvrage, la marée modifie la poussée d"Archimède sur la partie immergée, donc le poids apparent. Les contraintes verticales au sein du sol sousjacent varient donc de façon cyclique, au rythme de la marée, entre un maximum et un minimum. Il en va de même de la pression interstitielle dans un sol sousmarin.

Enfin, pour la construction des ouvrages la planification des travaux doit tenir compte de l'évolution du niveau de l'eau dans le temps.

Tout ceci justifie la prédiction du niveau de la mer avec un préavis approprié et une précision raisonnable. Or, depuis plusieurs décennies, les méthodes de prédictions de la marée n'ont pas connu d'améliorations sensibles, malgré les progrès technologiques des calculateurs.

Ces méthodes de prédictions reposent sur le développement du potentiel générateur de la marée, en une série de termes purement harmoniques (DOODSON 1954).

Des écarts algébriques significatifs sont souvent constatés entre cette prédiction et la réalité. Ces écarts constituent le phénomène physique, couramment appelé surcote.

La plupart des recherches menées actuellement pour cerner ce phénomène font référence à des méthodes déterministes par une modélisation de l'onde de tempête (FREROT, 1988 et MURTY, 1988). En revanche, notre étude vise à appréhender les surcotes pour un site donné (estuaire externe de la Loire) par une approche statistique, afin de mettre en lumière les paramètres les plus influents et de généraliser les lois qui en découleraient à un site quelconque. Dans cet article, nous présentons la méthodologie utilisée, ainsi que les premiers résultats que nous avons obtenus. 


\section{METHODOLOGIE}

Notre méthodologie s'articule suivant l'organigramme ci-dessous:

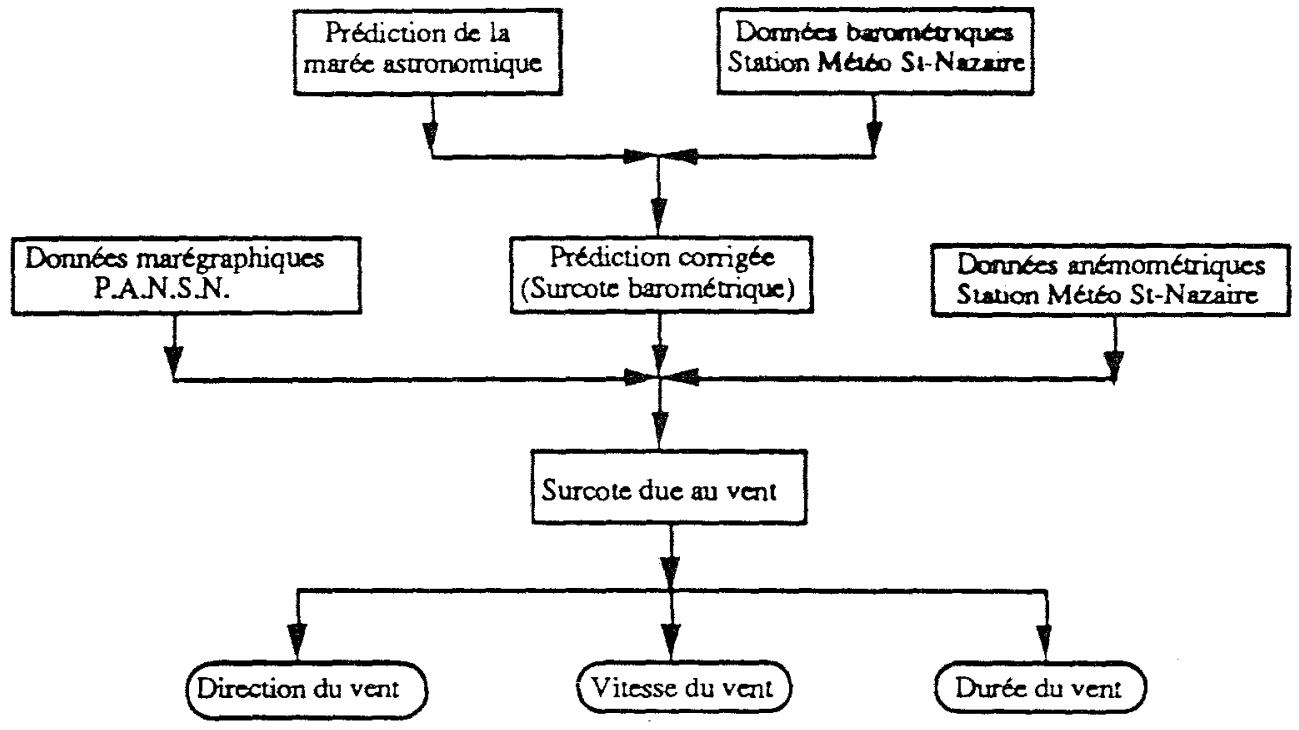

\section{2-1. Définition des surcotes.}

La surcote ou la décote en un point du littoral dépend des caractéristiques :

- propres au bassin océanique.

- de l'écoulement météorologique.

Soient $\mathrm{H}_{\mathrm{p}}(\mathrm{t})$ et $\mathrm{H}_{0}(\mathrm{t})$, respectivement la hauteur d'eau prédite par les calculs et la hauteur d'eau mesurée sur le site. On appelle surcote algébrique instantanée la quantité:

$$
S(t)=H_{0}(t)-H_{p}(t)
$$

\section{2-2. Calcul des surcotes}

Pour pouvoir calculer ces surcotes, il faut donc disposer d'une part d'une méthode de prédiction et d'autre part d'une série de mesures en nature.

Les mesures sont fournies par le marégraphe du port Autonome de Nantes-Saint Nazaire installé à Saint Nazaire, tandis que la méthode de prédiction par l'Analyse Harmonique qu'on a choisie repose sur le développement du potentiel générateur de la marée en une série de termes purement harmoniques (DOODSON 1954). Le niveau de la mer peut être ainsi modélisé par: 


$$
\begin{gathered}
\mathrm{H}_{\mathrm{p}}(\mathrm{t})=\mathrm{Z}_{0}+\sum_{j} \sum_{\mathrm{i}} \mathrm{A}_{\mathrm{ij}} \operatorname{Cos}\left(\mathrm{V}_{\mathrm{i} j}-\mathrm{G}_{\mathrm{i} j}\right) \\
\text { Ave. } \\
\begin{array}{cll} 
& \vdots & \text { l'indice de l'onde } \\
\mathrm{Z}_{0} & \vdots & \text { l'indice de la période de l'onde } \\
\mathrm{A}_{\mathrm{ij}} & \vdots & \text { l'amplitude de l'onde } \mathrm{i} \\
\mathrm{G}_{\mathrm{ij}} & \vdots & \text { la situation de l'onde } \mathrm{i} \\
\mathrm{V}_{\mathrm{ij}} & : & \text { l'argument astronomique de l'onde } \mathrm{i}
\end{array}
\end{gathered}
$$

L'argument astronomique de l'onde est la combinaison linéaire de six variables indépendantes (temps, longitudes des astres,...) dont les coefficients caractérisent les ondes élémentaires.

C'est cette méthode qui est développée dans le code de calcul de prédiction de la marée à St-Nazaire et qui prévoit la marée purement astronomique sans tenir compte des effets métérologiques (S.H.O.M. 1987).

Notre raisonnement repose sur l'hypothèse suivante : nous négligeons les erreurs et les incertitudes de la mesure et de la prédiction. Par conséquent, la différence $S(t)$ ne peut provenir que d'une origine météorologique.

\subsection{Surcote due à la pression atmosphérique}

Il est couramment appliqué une correction simple, statique. Cette correction équivaut à $1 \mathrm{~cm}$ par hPa en baromètre inversé (DARS et al. 1979).

\subsection{Etude de la surcote due au vent}

Une fois la correction de pression atmosphérique réalisée, la surcote résiduelle reste due au vent. En effet, dans son étude de l'influence des débits amont sur la prédiction de la marée dans la Loire Maritime, KHALIFA et al (1989) ont montré que ces débits n'ont pas d'influence à St-Nazaire.

Mais contrairement à la pression atmosphérique, il n'y a pas, a priori, de relation immédiate entre la surcote et le vent qui la provoque.

Notre approche statistique vise à mettre en parallèle, dans le site de SaintNazaire, d'une part les surcotes et d'autre part les mesures de vent. Les paramètres définissant le vent sont:

- la direction

- la vitesse

- la durée

Dans cette étude, nous essayons de dégager des corrélations entre les surcotes et les paramètres. Dans ce but, nous établissons un ensemble de courbes suivantes: 
- l'histogramme des fréquences des surcotes dues au vent.

- la rose des vents.

- les surcotes en fonction des directions du vent.

- les surcotes en fonction des vitesses du vent $\left(0^{\circ}\right.$ à $\left.360^{\circ}\right)$.

- les surcotes en fonction des vitesses du vent suivant les secteurs (de $0^{\circ}$ à $360^{\circ}$, tous les $45^{\circ}$ ).

Dans cette première analyse, nous n'avons pas tenu compte des durées du vent, qui sont pourtant très importantes. Sa prise en compte ultérieure, sera basée sur une estimation probabiliste du type du modèle ANKY (SPIRIDAKIS et al. 1990).

\section{PRESENTATION ET INTERPRETATION}

\subsection{Durée des mesures}

Pour un premier essai de notre méthodologie, nous avons traité trois mois de données : Janvier à Mars 1987. Les mesures sont issues du marégraphe de Saint-Nazaire. Le traitement des surcotes ne portera que sur les pleines mers et les basses mers.

\subsection{Surcotes calculées}

Pour cette période de trois mois nous avons calculé les surcotes, pour toutes les heures de pleine mer et de basse mer. Ces surcotes sont représentées dans l'histogramme de la figure 1. L'allure symétrique de cet histogramme fait penser à une distribution gaussienne, et par conséquent à un caractère aléatoire des surcotes.

\subsection{Influence de la direction du vent.}

La figure 2 représente ces surcotes. On remarque que pour cette période, les surcotes oscillent entre $\pm 60 \mathrm{~cm}$. Ceci correspond à l'ordre de grandeur auquel on s'attendait, compte tenu de la force du vent et du fait qu'il n'a pas été enregistré de tempête forte pendant les trois mois (vents inférieurs à $35 \mathrm{~km} / \mathrm{h}$ ). Il est difficile dans les circonstances de cette distribution d'interpoler une courbe moyenne. Dans l'ensemble, il y a plus de décotes que de surcotes. En revanche on constate que les vents soufflant d'un secteur compris entre $150^{\circ}$ et $270^{\circ}$ (SSE à $W$ ) entraînent en moyenne une légère surcote. Les autres vents entraînant en moyenne des décotes. 


\subsection{Influence de la vitesse.}

La rose des vents représentée en figure 3 montre que pour cette période:

- les vents dominants soufflent d'un secteur NNE-ENE.

- ie vent existe de façon non négligeable dans toutes les directions sauf le secteur Sud à Est.

La représentation des surcotes en fonction des vitesses de vents (figure 4) ne révèle pas une justification de la surcote par la vitesse instantanée du vent. Ceci est très probablement dû au fait qu'une surcote est provoquée par un vent établi depuis plusieurs heures.

Néanmoins, l'étude des vitesses de vents, uriées par plages de direction montre dans le cas particulier du secteur S-SW (figure 5) une absence de surcotes ou de décotes pour les vents de moins de $5 \mathrm{~m} / \mathrm{s}$. Cependant cette relation ne peut pas être généralisée. En effet, nous trouvons souvent des vents nuls entraînant de fortes surcotes ou décotes. Ceci s'explique par l'importance de la durée du vent, l'effet de surcote engendré par un vent peut persister, après que ce vent ait cessé de souffler.

Dans une phase ultérieure, cette notion de durée sera mise en évidence. Notre base de données anénométrique devra être enrichie par l'interpolation des durées de chaque vent correspondant à une pleine mer ou une basse mer. De même que la durée d'établissement d'une surcote donnée sera mise en lumière.

\section{CONCLUSIONS ET DEVELOPPEMENT}

Dans cet article, nous présentons la méthodologie de travail que nous avons mise au point ainsi que les outils théoriques (modèle de prédiction de la marée) et expérimentaux (données baramétriques, anémonétriques et marégraphiques) nécessaires à l'étude des surcotes de marée dans le site de l'estuaire de la Loire. Ces premiers résultats ne sont qu'une esquisse de cette étude de surcote. Des développements s'imposent, pour aboutir à des résultats définitifs.

Les applications exposées ici, ne portent en effet que sur une période de trois mois. Or, notre approche statistique du phénomène nécessite lá prise en compte d'une grande période de plusieurs années.

L'existence d'une telle série de mesures anémométriques faiblement espacées dans le temps (quelques années) permettra de déduire les durées de vent, qui ont une influence importante sur les surcotes. Il deviendra alors possible d'isoler d'une part les coups de vents (tempêtes) et d'autre part les vents établis et d'analyser leurs effet respectifs sur les surcotes. 
La prise en compte des durées de vent permettra également d'éffectuer leur corrélation avec les durées de surcotes établies.

\section{REFERENCES}

PORT AUTONOME DE NANTES-SAINT NAZAIRE; Annuaire des marées ; Nantes; 1987.

DARS M.; BESSERO G.; GUENVEL D.; "Cours de marée"; Tome 1; Ecole Nationale Supérieure de Techniques Avancées; Paris; 1979.

DOODSON A. T.; "Développement harmonique du potentiel générateur de la maree"; Revue Hydrographique Internationale; pp: 29-61; 1954.

FREROT O.; "Description d'un modèle déterministe de prévision des surcotes en Manche et Mer du Nord (1)"; Annales des Ponts et Chaussées; No: 45; pp: 20-32; Paris; 1988.

MURTY T. S.; "Mathematical modelling of global storm surges problems"; Natural and man-mode hazards; International Symposium; pp: 239-255; Edit: D. Reidel publishing Company; 1988.

O.A. KHALIFA; RIOU Y. ; THOMAS P.; "Contribution d l'étude des surcotes dans la Loire: Influence du débit fluvial"; Rapport de contrat; ENSM ; Nantes ; 1989.

SER VICE HYDROGRAPHIQUE ET OCEANOGRAPHIQUE DE LA MARINE (S.H.O.M.); "Table des marées des grands ports du monde"; Paris; p: 186; 1987.

SPIRIDAKIS M.; KOZIS L.; "Calcul automatique des caractéristiques de la houle générée par le vent dans les mers Grecques: Le modèle ANKY"; Symposium Littoral 90; pp: 186-190; Marseille; 1990. 


\section{SESSION I}

Figure 1 : Histogromme des surcotes

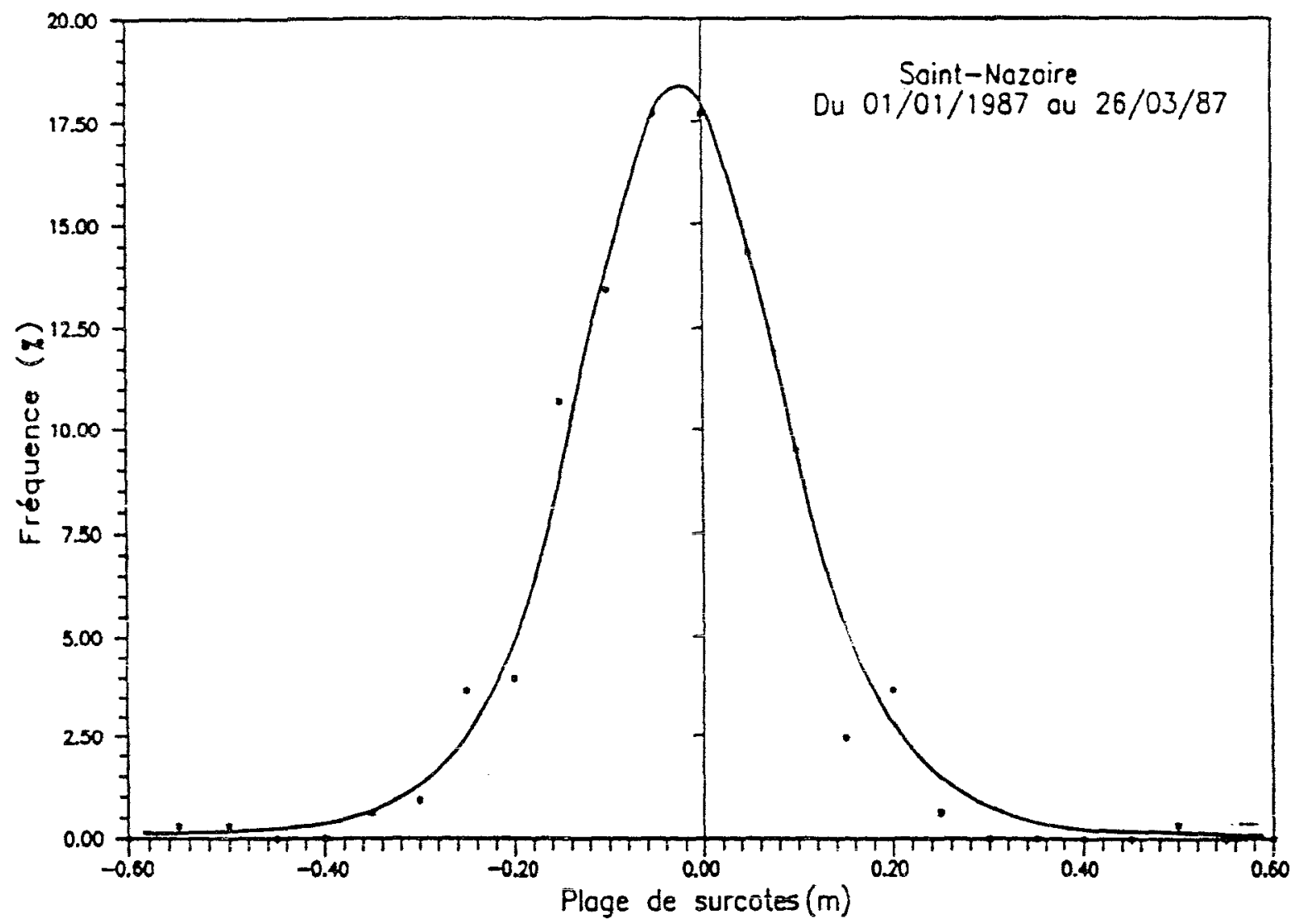

Figure 2: Correlation entre les surcotes et les Directions du Vent

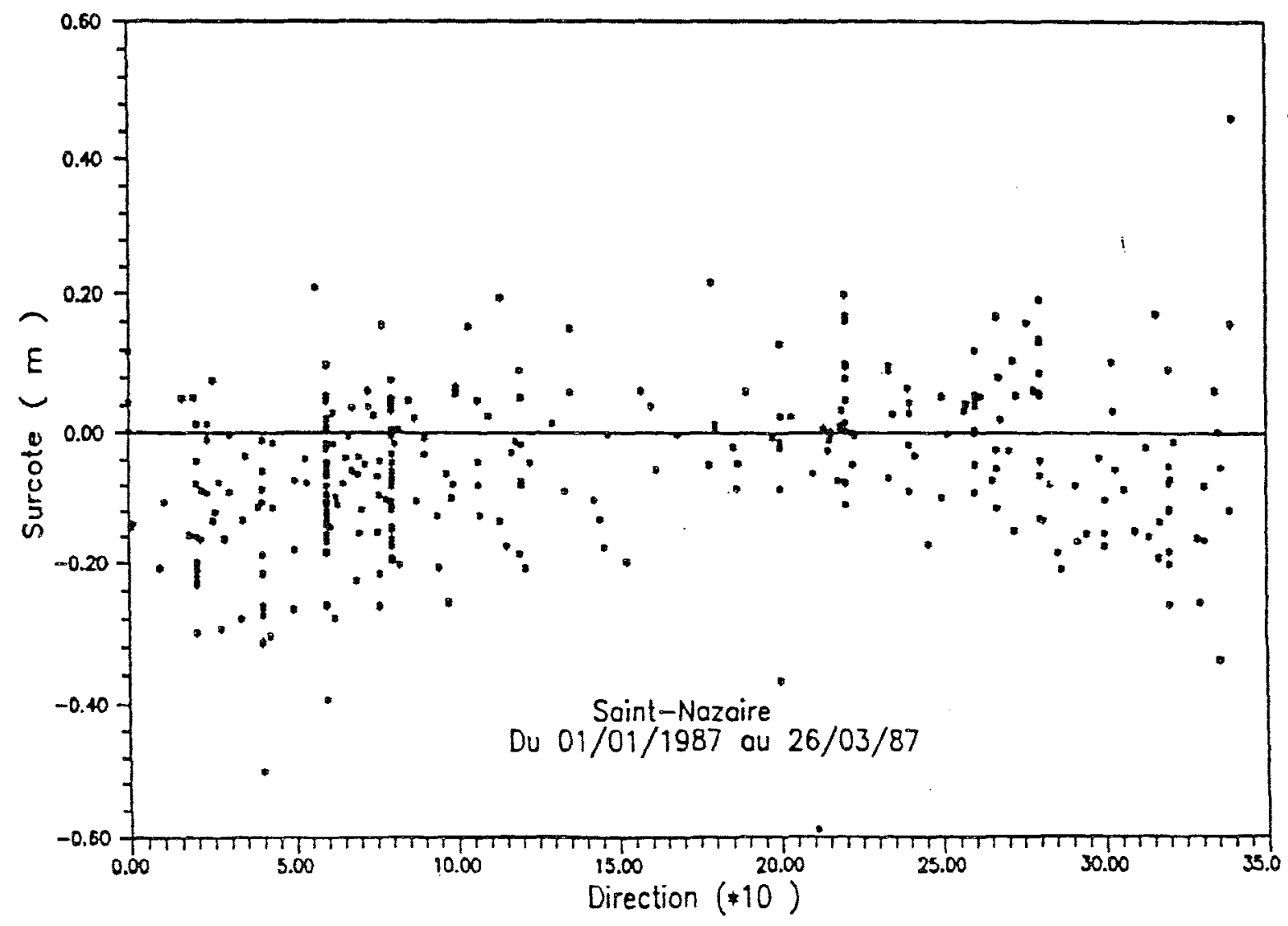




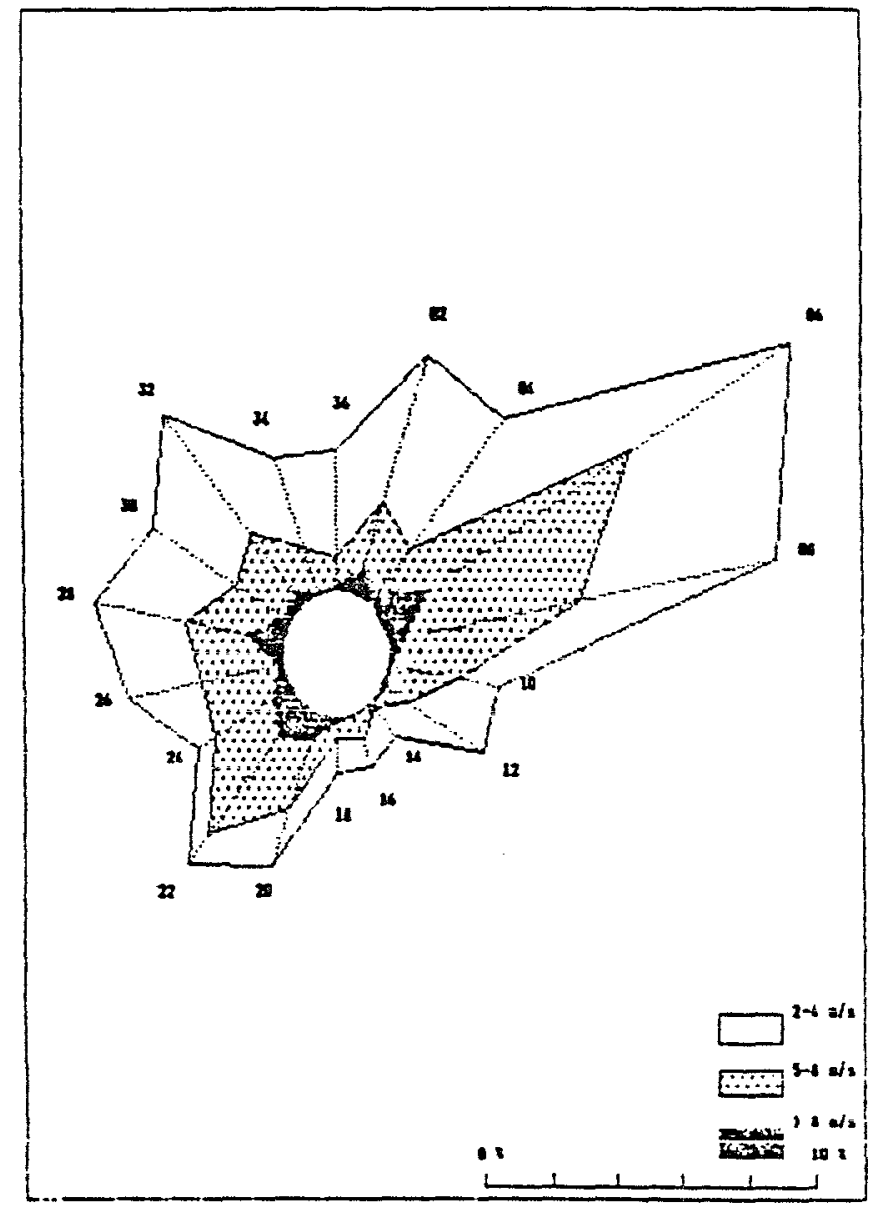

Figure 3 : Fréquences moyennes des directions du vent à St-Nazaire $01 / 01 / 87$ au $26 / 03 / 87$ 
Figure 4: Correlation entre les surcotes et les Vitesses du Vent

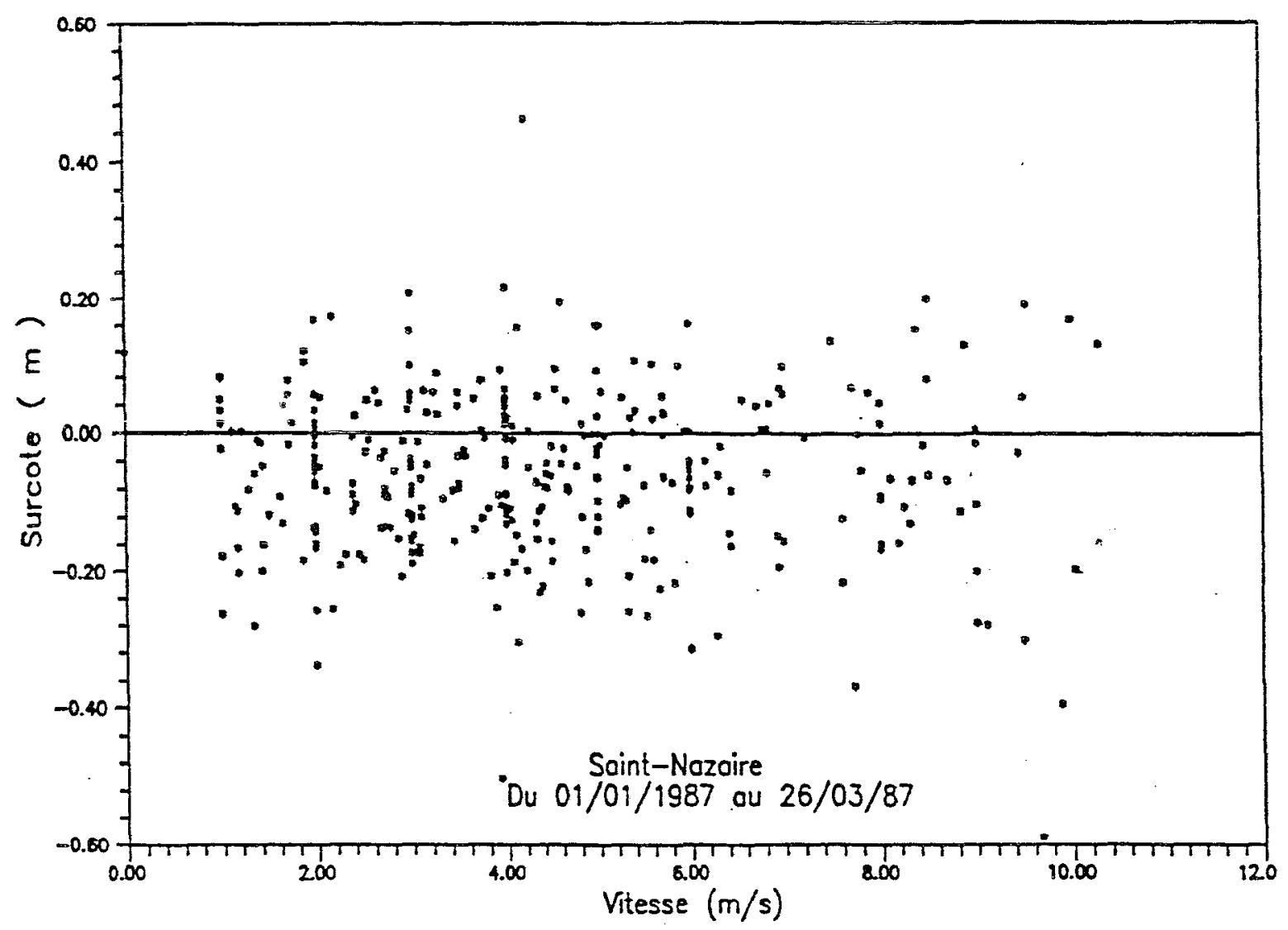


Figure 5: Correlotion entre les surcotes et les Vitesses du Vent pour la plage 180 a 225

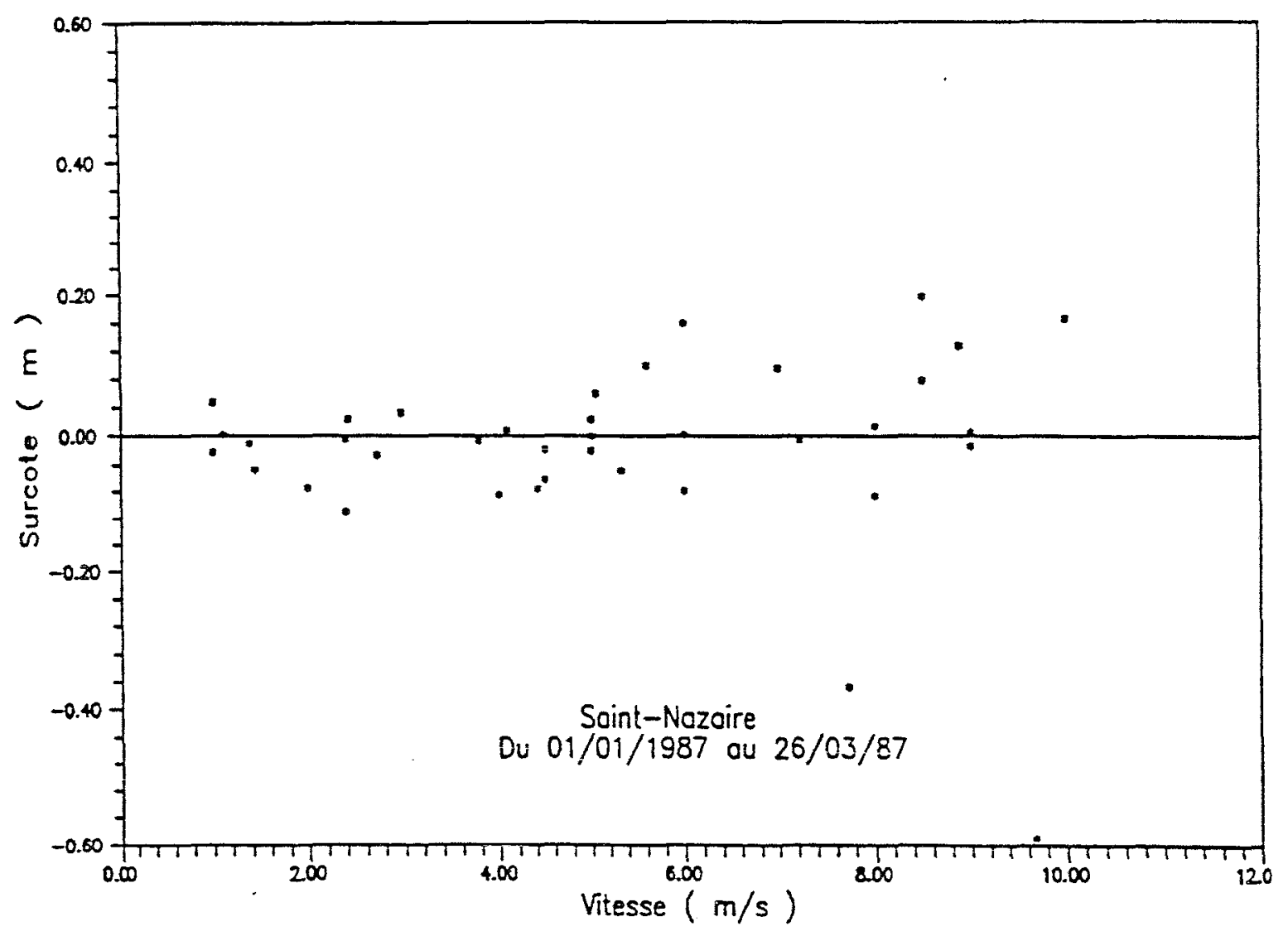

